\title{
Multiple Wavelength LED on Monolithic QW Structure
}

\author{
Abdullah J. Zakariya*a,b,c Patrick LiKamWa ${ }^{\text {a,b }}$ \\ ${ }^{\mathrm{a}} \mathrm{CREOL}$, The College of Optics and Photonics \\ ${ }^{b}$ Department of Electrical Engineering and Computer Science \\ University of Central Florida, 4000 Central Florida Blvd., Orlando FL 32816 \\ ${ }^{\mathrm{c}}$ Information Technology \& Communication Sector, MOI-Kuwait \\ P.O. Box 1250, Safat 71655, Kuwait \\ *azakariya@knights.ucf.edu
}

\begin{abstract}
A monolithically integrated multi-wavelength LED based on selective dielectric cap intermixing is investigated experimentally. The proposed LED emits radiation with multiple wavelength peaks from one compact easy to fabricate quantum well $(\mathrm{QW})$ structure. Each wavelength has an independent emission power control, allowing the LED to radiate one or more wavelengths simultaneously. The LED material is an AlGaAs/GaAs QW p-i-n heterostructure. The device is divided into three selectively intermixed regions using an impurity-free vacancy induced intermixing technique creating localized intermixed areas. Each region is intermixed to varying extent resulting in different luminescence peaks and by separately addressing each section with its electrical current, the net emission spectrum can be fully controlled. The fabrication process starts with the growth of a $400 \mathrm{~nm}$ thick layer of $\mathrm{SiO}_{2}$ over the whole sample using plasma enhanced chemical vapor deposition. Three regions with different $\mathrm{SiO}_{2}$ thicknesses are defined via two photolithographic and subsequent reactive ion etching steps. The sample is then annealed at $975^{\circ} \mathrm{C}$ for $20 \mathrm{~s}$ to activate the intermixing of the constituent atoms of the quantum well and barrier materials. The degree of intermixing is determined by the thickness of the $\mathrm{SiO}_{2}$ cap. After removal of the $\mathrm{SiO}_{2}$ cap, contact stripes are evaporated on each region to act as an independent intensity power control for that region. Experimental results have shown that a controllable $10 \mathrm{~nm}, 21 \mathrm{~nm}$ and $33 \mathrm{~nm}$ blue shifts of the peak wavelength of emission from that of the as-grown sample corresponding to $0,100 \mathrm{~nm}$, and $400 \mathrm{~nm}$ thick $\mathrm{SiO}_{2}$ caps respectively.

Keywords: Impurity intermixing, quantum wells, LEDs, multi-wavelength.
\end{abstract}

\section{INTRODUCTION}

Light Emitting Diodes (LEDs) have recently become a target of the electronics industry due to its versatility and applications that ranges from everyday uses to medical uses in today's technology. LEDs have shown promising results in medical research where it is used in monitoring blood pressure, hair therapy and dermatology $y^{1,2}$. Furthermore, LEDs are also being used in archeological research to provide a non-invasive sample probing $\mathrm{g}^{3,4}$. In addition, LEDs are widely marketed as a compliant green technology too. LEDs allow lower power consumption, have longer lasting life, and give better heat dissipation. They provide a brighter display, with thinner and smaller panels, as well as better contrast levels. Commercial multicolor LED products are in high demand and have already taken over as the main components for light fixtures and digital TVs. Red, Green and Blue (RGB) LEDs are now used mainly as backlights for high end displays and monitors, but size and fabrication complexity in producing multicolor LEDs are still main challenges that the industry has to overcome. Methods were developed to overcome the size challenge where devices with multiple emission wavelengths are fabricated by growing two different MQWs on a single structure ${ }^{5,6}$. Nano-columns emitting LEDs that emit light blue to red have shown promising results ${ }^{7}$. Although these devices are small in size, packaging and integration contribute to about $80 \%$ of the $\operatorname{cost}^{8}$ and they require extensive and complex fabrication. Moreover, the realization of RGB-LED white lighting has several challenges. In white light applications LEDs vary in lumen output and wavelength, and in their dependence on light temperature. Since mixing one or more red, green and blue LEDs is creating white light, variation in one color (or one LED) can have a significant effect on the color point.

We propose a single output monolithic LED shown in Fig.1 that has the ability to shift wavelength in visible color spectrum. The proposed LED contains three regions with selectively intermixed quantum wells (QW) of varying degrees. Each region would emit one of the components of RGB colors that would be independently controlled.

Twelfth International Conference on Solid State Lighting and Fourth International Conference on White LEDs and Solid State Lighting, edited by Matthew $\mathrm{H}$. Kane, Christian Wetzel, Jian-Jang Huang, lan T. Ferguson,

Proc. of SPIE Vol. 8484, 84840T - @ 2012 SPIE · CCC code: 0277-786/12/\$18 - doi: 10.1117/12.928011 
By combining red, green and blue we can produce white light, and by adjusting the ratios of the three colors independently we can produce a wide range of colors. The design of the multi wavelength structure allows for monolithically integrating LED pixels with different wavelengths. The proposed LED consists of a uniformed monolithic structure that is annealed in one shot; therefore, the uniformity of colors and intensities is given. This approach can also be used as on on-chip monolithic multiplexer allowing an easy integration of on board communication devices which results in lower packaging costs.

In this work we demonstrate the feasibility of the proposed idea by fabricating a monolithically integrated single output multi-wavelength LED based on selective area intermixing of a QW structure. The LED emits radiation with dual wavelength peaks from one compact monolithic quantum well $(\mathrm{QW})$ structure and is fabricated using only two lithographic fabrication steps. Each wavelength has an independent emission power control, allowing the LED to radiate one or more wavelengths separately or at once. Unlike other monolithic multi wavelength LEDs where more than one MQW structures are grown on a substrate, our device uses only one single QW structure throughout the device and can be as small as $400 \mu \mathrm{m}^{2}$.

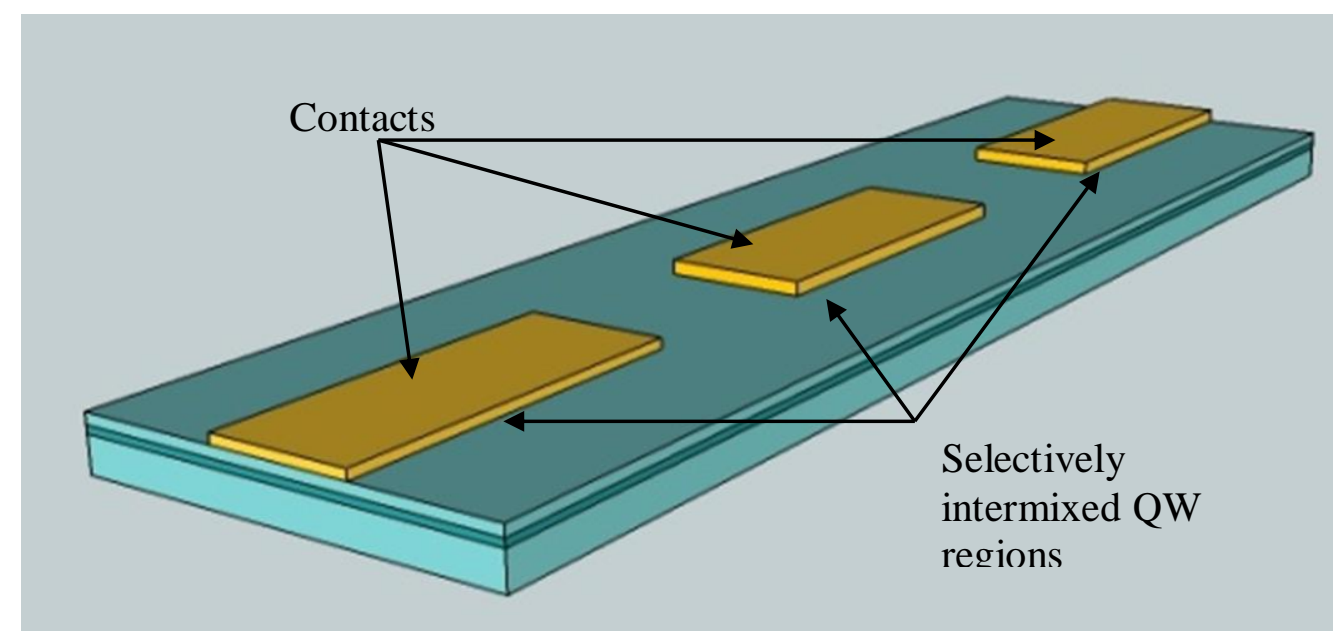

Fig.1 Proposed single output multi-wavelength LED

\section{METHODOLOGY}

\subsection{Theory and Device design}

For this work, a simpler prototype device is fabricated first to determine the feasibility of the proposed device and to obtain preliminary results and observations. The main principle of operation for this LED depends on quantum well intermixing. The intermixing method used in this work is impurity-free vacancy diffusion (IFVD). This technique offers a cost effective, area selective and highly reproducible method ${ }^{7}$ of realizing different optical bandgaps on one single substrate. The areas that are designated to be intermixed are capped with $\mathrm{SiO}_{2}$ film and the sample is annealed at a sufficiently elevated temperature for a very short duration. Different peaks in the emission spectra can be achieved by introducing different $\mathrm{SiO}_{2}$ film thicknesses ${ }^{9}$. During the high temperature annealing process, the $\mathrm{Al}$ atoms diffuse into the quantum well region and the abruptness of the interfaces is destroyed causing the sub-band energies to move apart, thereby resulting in an increase in the $n=1$ electron to heavy-hole transition energy ${ }^{9}$. The device consists of three selectively intermixed regions of different $\mathrm{SiO}_{2}$ film thicknesses.

Ultimately the choice of substrate material and quantum well intermixing characteristics will determine the wavelength of operation and the range of wavelength shift. We can controllably tune the wavelength peak of the gain spectrum by a blue shift as a result of $\mathrm{SiO}_{2}$ capping film on top of the MQW structure which promotes the diffusion of $\mathrm{Ga}$ into that cap resulting in vacancies being formed in the semiconductor structure immediately below the surface. During rapid thermal annealing the vacancies are driven through the MQW structure randomly exchanging lattice sites with $\mathrm{Ga}$ and $\mathrm{Al}$ especially at the quantum well to barrier interfaces. The gentle grading of the energy potential give rise to an increase in the eigen energy levels of the confined electrons and an equivalent 
decrease in the eigen level for holes thereby leading to an effective band gap increase ${ }^{10}$. Depending on the MQW structure and the deposited capping material, either an enhanced blue shift or a suppressed of shifting of the emission peak has been detected by photoluminescence (PL) measurements. Moreover, it has also been reported that plasma exposure can reduce the diffusivity of $\mathrm{Ga}$ into the capping layer due to the changing properties and porosity ${ }^{11}$. In this experiment, the intermixed regions are $20 \mu \mathrm{m}$ wide by $200 \mu \mathrm{m}$ long covered by p-type electrical contacts and separated by $50 \mu \mathrm{m}$. Electrical current can therefore be independently injected into the separate sections allowing full control of the net emission spectrum of the output light.

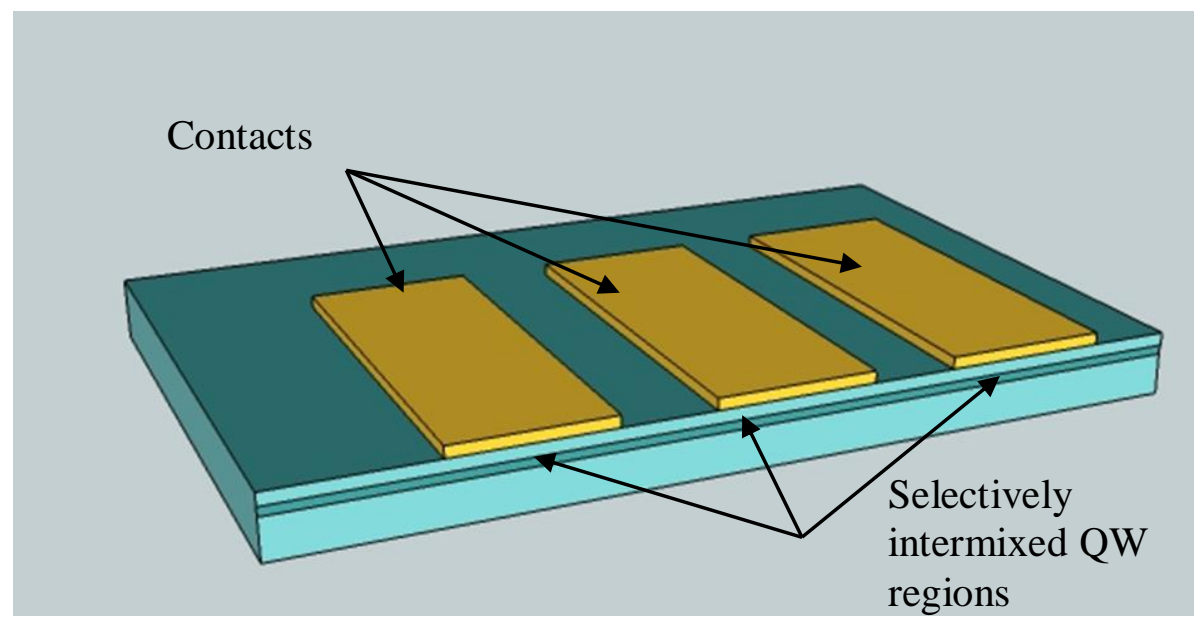

Fig.2. System overview showing the fabricated test LED device

\subsection{Fabrication}

In this work we designed and fabricated the LED on a GaAs/AlGaAs QW graded index separate confinement heterostructere (GRINSCH) p-i-n material that has an as-grown electroluminescence peak of $805 \mathrm{~nm}$.

The choice of material is based on the optical and electrical properties and ease of fabrication and its compatibility with relatively straight-forward fabrication techniques. The wafer's active region consists of a single $4 \mathrm{~nm}$ wide GaAs quantum well sandwiched between two $5 \mathrm{~nm}$ wide $\mathrm{Al}_{0.25} \mathrm{Ga}_{0.75} \mathrm{As}$ barrier layers. These layers are surrounded by $\mathrm{AlGaAs}$ layers each $150 \mathrm{~nm}$ wide, where the concentration of aluminum is gradually increased from $\mathrm{Al}_{0.25} \mathrm{Ga}_{0.75} \mathrm{As}$ to $\mathrm{Al}_{0.55} \mathrm{Ga}_{0.45} \mathrm{As}$. Those layers are further sandwiched by $\mathrm{Al}_{0.55} \mathrm{Ga}_{0.45} \mathrm{As}$ upper and lower cladding layers that are each $1.2 \mu \mathrm{m}$ wide. The top of the wafer is capped off by a $50 \mathrm{~nm}$ transition layer followed by $150 \mathrm{~nm}$ GaAs layer.

Four photolithographic masks for the device fabrication process were designed and cut in-house using a NEOS laser writer. The first mask is the alignment marks mask, which is also repeated throughout the rest of the masks. The second and third masks define the windows for the intermixed regions. Finally, the fourth mask is the P-type contacts mask.

The fabrication process starts by exposing through the first mask to pattern the alignment marks followed by chromium evaporation. The chromium alignment marks are repeated throughout the subsequent masks to serve as fiduciary markers for the various photolithographic steps. A $400 \mathrm{~nm}$ thick layer of $\mathrm{SiO}_{2}$ layer is deposited by plasma enhanced chemical vapor deposition (PECVD) over the whole sample. The second mask is used with negative photoresist and reactive ion etching (RIE) to create the first window that defines the first intermixed region. The first RIE step is used to etch down the $\mathrm{SiO}_{2}$ to a $200 \mathrm{~nm}$ thickness. Using similar photolithographic process with mask three followed by subsequent reactive ion etching step, windows are opened in the $\mathrm{SiO}_{2}$ film to define the uncapped regions.

The sample is then annealed at $975^{\circ} \mathrm{C}$ for 20 s to activate the intermixing of the constituent atoms of the quantum well and barrier materials via dielectric capped vacancy induced quantum well disordering ${ }^{12}$. The degree of intermixing is determined by the thickness of the $\mathrm{SiO}_{2}$ cap. The sample is then immersed in Buffered Oxide Etch (BOE) to remove the $\mathrm{SiO}_{2}$ cap off the whole sample. The fourth mask is then exposed and the top P-type contacts 
for $\mathrm{Ti}, \mathrm{Zn}$ and $\mathrm{Au}$ metals are evaporated over the three regions through photo-lithographically defined windows in negative photoresist. After lift-off of the unwanted metals, three electrical contacts remain, one on top the originally uncapped region, one on top the originally $200 \mathrm{~nm} \mathrm{SiO}_{2}$ cap and one on top of the intermixed region originally capped by $400 \mathrm{~nm}$ of $\mathrm{SiO}_{2}$ to act as independent intensity power controls. The final dimensions of the device consisted of three parallel contacts of $20 \mu \mathrm{m}$ wide and $1 \mathrm{~mm}$ separated by $100 \mu \mathrm{m}$.

\section{EXPERIMENTAL RESULTS}

\subsection{Electroluminescence}

To determine the required amount of intermixing needed to emit three wavelength peaks from the LED, the degree of disordering was measured by comparing the electroluminescence for different samples with different $\mathrm{SiO}_{2}$ caps. Several samples were prepared for this part of the testing. The first sample was simply the as-grown sample. The second batch of samples consisted of an uncapped sample and four other samples that had $\mathrm{SiO}_{2}$ deposited films of $100 \mathrm{~nm}, 200 \mathrm{~nm}, 300 \mathrm{~nm}$ and $400 \mathrm{~nm}$ which were all annealed at $975^{\circ}$ for $20 \mathrm{~s}$. P-type contacts were evaporated on top and defined by lift-off, and then the samples were thinned down and n-type contacts were evaporated on the bottom. To obtain the electroluminescence spectra, samples were mounted on copper headers and electrical current is injected into the device using a micro-positioner probe. The optical output from each sample was collected by a 20x lens and focused simultaneously into a spectrometer and a CCD camera as shown in Fig.3.

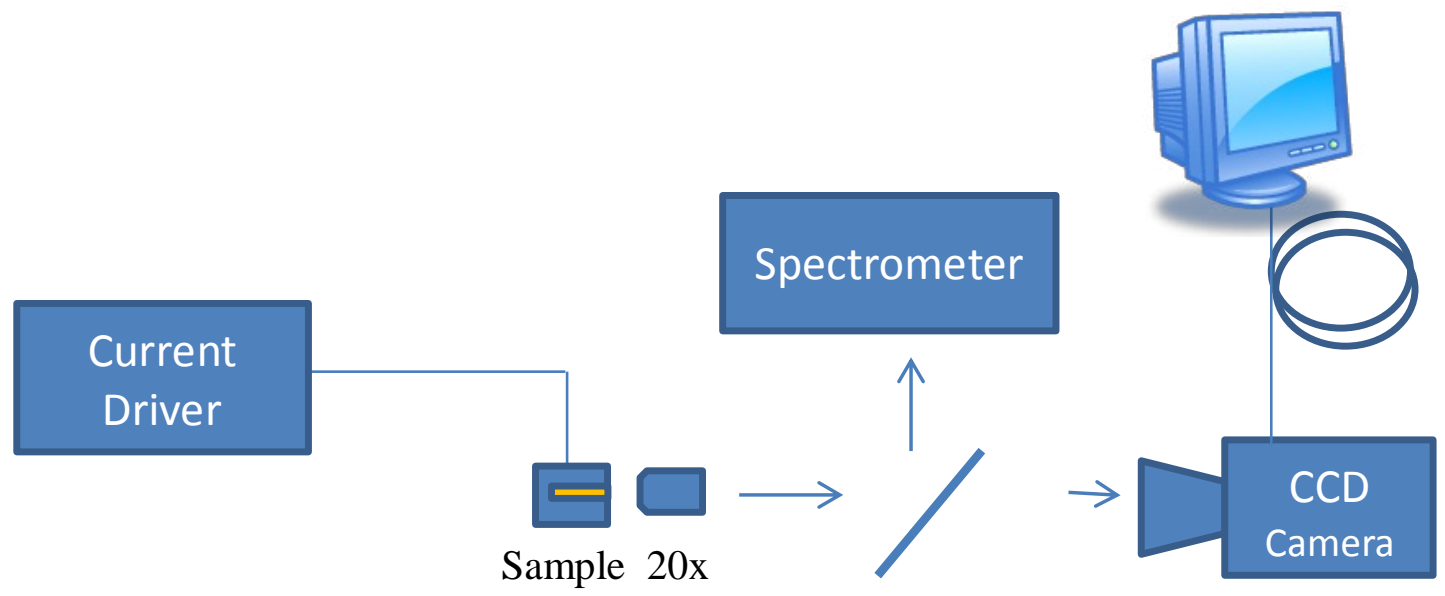

Fig.3 Electroluminescence experimental set-up

The electroluminescence measurements indicate that the QW intermixing using this method results in a controlled $10-33 \mathrm{~nm}$ blue shift of the effective band edge of the coated samples compared to a shift of $5 \mathrm{~nm}$ for the uncoated samples. Fig.4 shows the measured spectra of the tested samples.

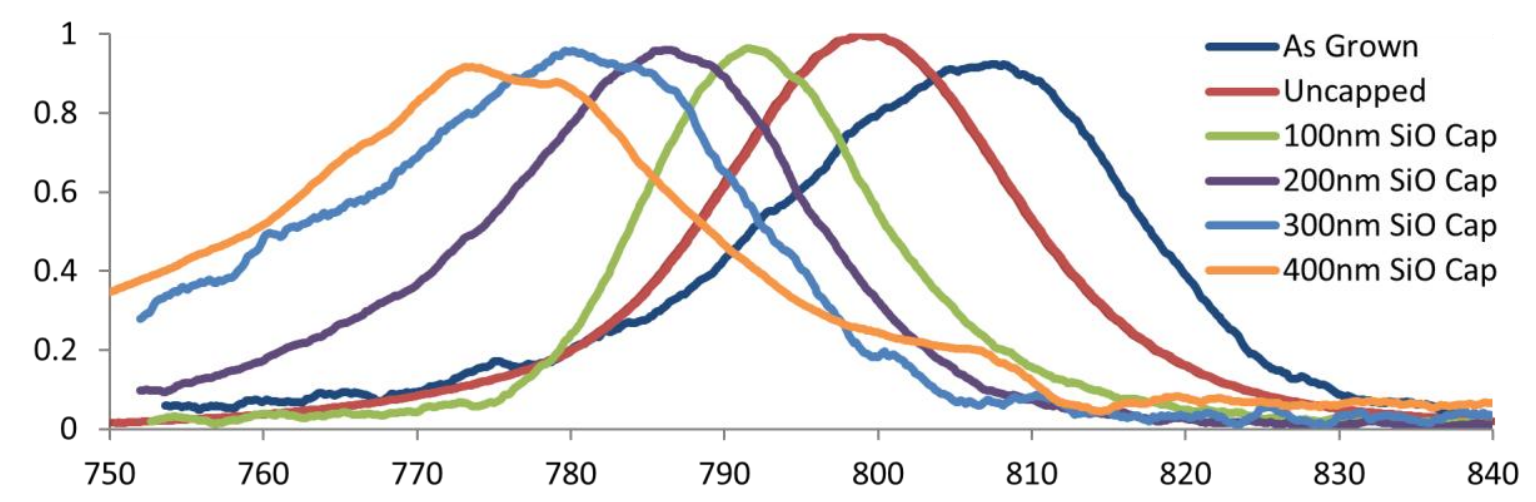

Fig. 4 shows the electroluminescence measurement for different $\mathrm{SiO}_{2}$ capped thicknesses QW samples. 


\subsection{LEDs Testing and Results}

The devices are cleaved at both end-facets for a total length of $1 \mathrm{~mm}$ and their performance is investigated using three separate current drivers to inject currents to the contact stripes via micropositioner probes. Currents are applied to each contact separately, then to two contacts and finally to all three contacts simultaneously. When current is applied, the generated electroluminescence propagates in the slab waveguide. The outputs of the device are collected by a 20x lens and focused on a spectrometer. The outputs from the edge facet and that from the device top surface are simultaneously monitored by two CCD cameras that are connected to a digital frame grabber as shown in Fig.5. The device was mounted such that all of the three disordered area contacts face the lens. Therefore to collect the emitted wavelength, the lens had to be moved accordingly to collect the emitted light.

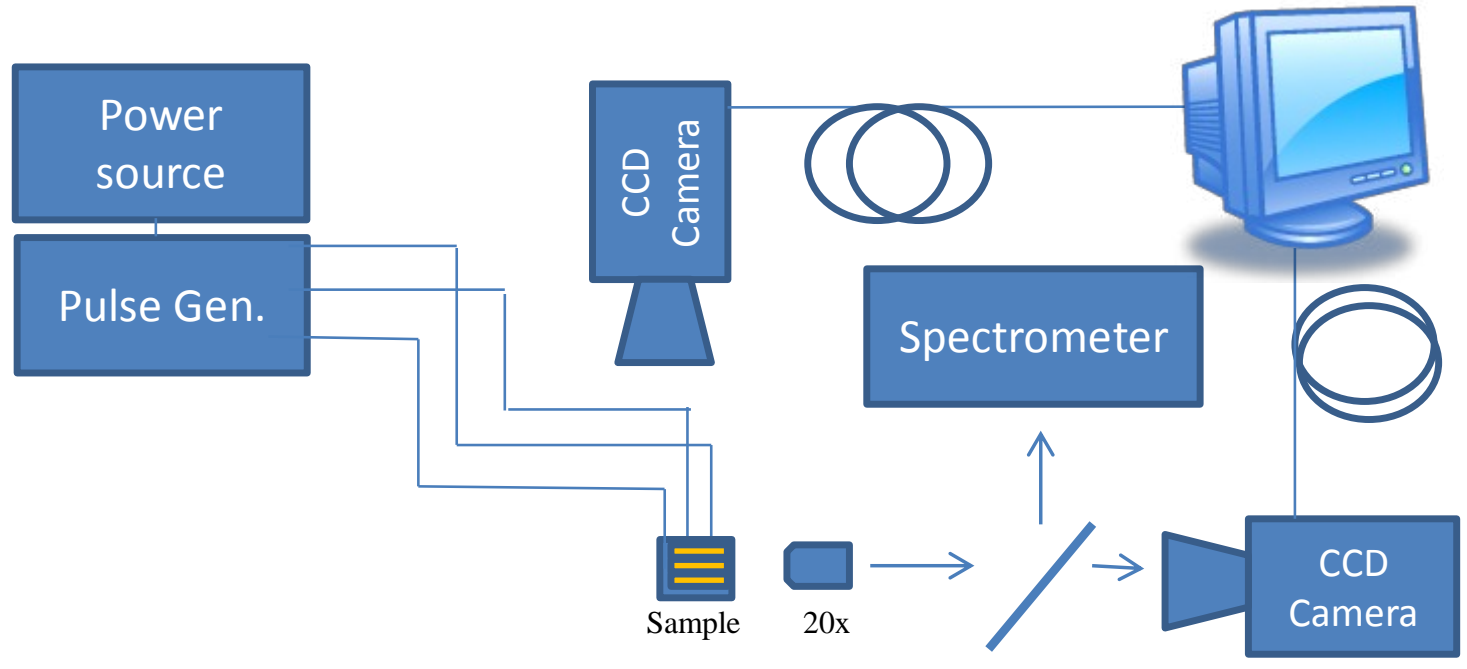

Fig.5 LED's experimental set-up

Experimental results show that the peak emission from the originally uncapped annealed region is blue shifted by $5 \mathrm{~nm}$ from the peak wavelength of the as-grown sample. On the other hand, a controllable blue shift of the peak wavelength of emission from the $\mathrm{SiO}_{2}$ capped region depending on the thickness of the $\mathrm{SiO}_{2}$ film is achieved. A recorded $28 \mathrm{~nm}$ and $16 \mathrm{~nm}$ blue shift for the $400 \mathrm{~nm}$ and $200 \mathrm{~nm} \mathrm{SiO}_{2}$ thick is obtained. Fig.6(a) shows the spectra obtained from the primary edge output facet of the device with current injected to the contacts separately. Fig.6(b) shows sequence pictures of the top view of the device with different combinations of current applied to two contacts of the device. An optical power meter placed in the output beam path recorded an optical power of $11.1 \mathrm{~mW}$, $11.2 \mathrm{~mW}$ and $11 \mathrm{~mW}$ for the $400 \mathrm{~nm}$ capped, $200 \mathrm{~nm}$ capped and the uncapped regions respectively at $50 \mathrm{~mA}$ current injection which resulted in a calculated responsivity of $\approx 0.22$ W/A.

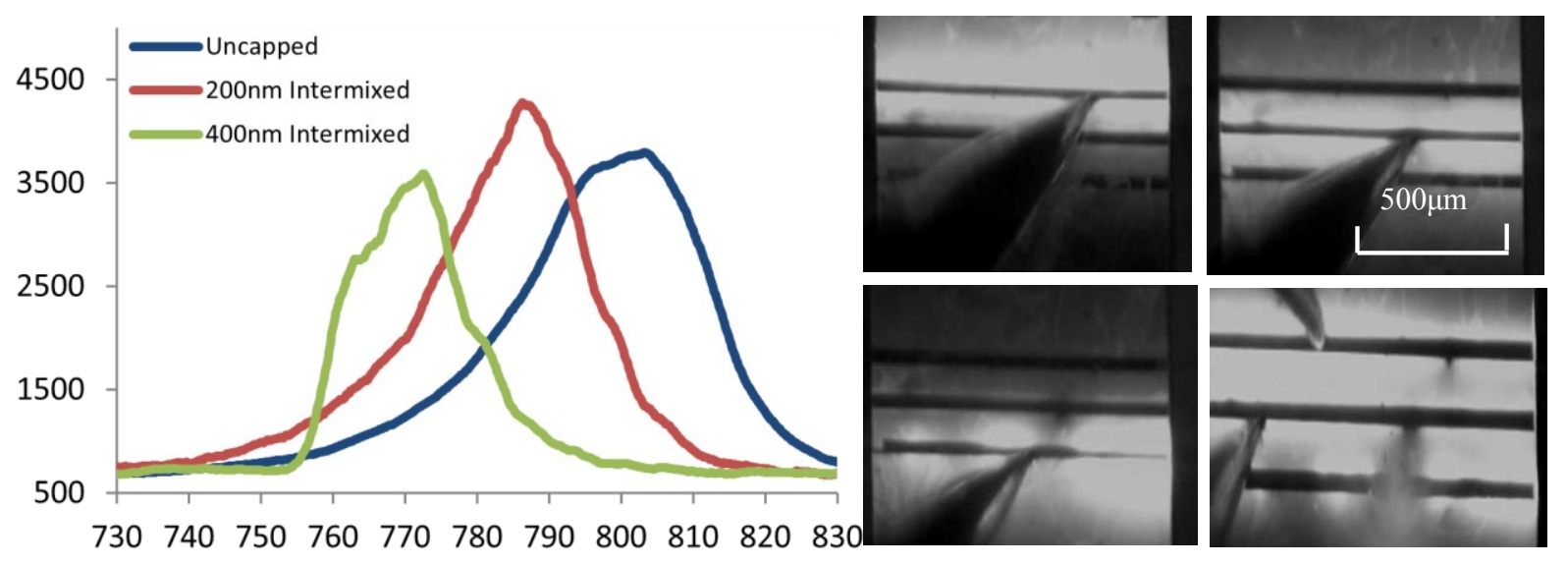

Figure 6 (a) Device output spectra (b) Device sequence pictures of applied currents for different regions 


\section{CONCLUSION}

In conclusion, we have fabricated and experimentally investigated a single output multi wavelength monolithic LED device based on a selectively intermixed GaAs/AlGaAs QW laser structure. Wavelengths intensities are independently controlled by three separate current sources. This approach shows the ability to emit three wavelengths from one structure simultaneously or separately with different intensities. The electroluminescence results indicate that the QW structure has the ability to controllably emit $800 \mathrm{~nm}, 789 \mathrm{~nm}$ and $772 \mathrm{~nm}$ either simultaneously or separately. We are confident that a monolithic multicolor RGB LED can be fabricated based on the same principle shown here.

\section{FUTURE WORK}

The proposed design is going to be adapted on to the next device; however, the device material will be selected such that the intermixed regions emit RGB color. Moreover, a proposed QW substrate with a narrower QW active core is to be used. The optical bandgap can be engineered to such that each selectively intermixed region emits a different color. In addition, a substrate etched waveguide that leads light in the active layer will be introduced to ensure light directionality and the ability to have a focused output.

\section{ACKNOWLEDGMENTS}

Abdullah Zakariya would like to acknowledge the financial support from the Ministry of Interior - Kuwait: Information Technology and Communication Sector, the Kuwait Foundation for the Advancement of Science (KFAS) and the support of Assistant Undersecretary of Information Technology and Communication Shiekh/ Mishal Al-Jaber Al-Abdullah Al-Sabah. The authors would also like to acknowledge the technical discussion and the help provided by Dr. Daniel May-Arrioja.

\section{REFERENCES}

[1] Daniel Barlot,"Light Emitting Diodes (LEDs) in Dermatology," semin in cutan med and surg, Elsevier 27, 227-238 (2008)

[2] Ammar Al-Ali "Blood Parameter Measurement System," US Patents 7,027,849 B2. (2006).

[3] Haida Liang, Borislava Peric, Michael Hughes, Adrian Podoleanub, Marika Spring, David Saundersd," Optical Coherence Tomography for Art Conservation \& Archaeology," Proc. SPIE 6618, 661805-1 (2007)

[4] N. Spooner, M. Aitken, B. Smith, M. Franks and C. McElroy," Archeological Dating by Infrared Stimulated Luminescence using a Diode Array," Radiation Protection Dosimetry. Vol.34, No.114, pp. 83-86, (1990)

[5] Seungyong Jung, Sergey Suchalkin, Gela Kipshidze, David Westerfeld, Eric Golden et al, "Dual wavelength GaSb based type I quantum well mid-infrared light emitting diodes,” Appl. Phys. Lett. 96, 191102 (2010)

[6 Michael J. Grundmann, Umesh K. Mishra," Multi-color light emitting diode using polarization-induced tunnel junctions," phys. stat. sol. (c) 4, No. 7, 2830-2833 (2007)

[8] MIT Microphotonics Center Industry Consortium," Microphotonics: Hardware for the Information Age Executive Overview," Microphotonics Center at MIT, Cambridge, MA. Rep. 2005

[9] X. F. Liu, B. C. Qiu, M. L. Ke, A. C. Bryce, and J. H. Marsh, "Control of Multiple Bandgap Shifts in InGaAs-AlInGaAs Multiple-Quantum-Well Material Using Different Thicknesses of PECVD SiO2 Protection Layers," IEEE Photonics Technology Letters, VOL. 12, NO. 9, (2000).

[10] D. A. May-Arrioja, N. Bickel, P. LiKamWa, "Integrated beam-steered optical switch," Proc. SPIE 5435, 91-96 (2004).

[11] A. Saher Helmy, S. K. Murad, A. C. Bryce, J. S. Aitchison, J. H. Marsh, S. E. Hicks, and C. D. W. Wilkinson, "Control of silica cap properties by oxygen plasma treatment for single-cap selective impurity free vacancy disordering," Applied Physics Letters, VOL. 74, NO. 5, (1999).

[12] C. Kim, D.A. May-Arrioja, P. LiKamWa, P. Newman, J. Pamulapati, "Ultrafast all-optical multiple quantum well integrated optic switch,” Electronics Lett., 36,(23), pp1929-1930, (2000) 